\title{
Context Awareness Technology Based on Ontology Mechanism for Sensor Networks
}

\author{
Biao Dong ${ }^{1, a}$, Jinhui Chen ${ }^{2, b}$
}

${ }^{1}$ School of Computer \& Software, Nanjing Institute of Industry Technology, Nanjing, 210023, China

${ }^{2}$ School of Computer \& Software, Nanjing University of Information Science \& Technology, Nanjing, 210044, China

aemail:dongb@niit.edu.cn, bemail:cjh@nuist.edu.cn

Keywords: Context Awareness; Ontology; Sensor Networks

Abstract. This paper presents a semantic-oriented approach for architecting sensor networks applications using ontology mechanism. Considering the validity and the timeliness which are necessary for sensor networks knowledge sharing and reusing, a common and extensible context ontology model is defined to describe the related concepts and their relationships. A context awareness process model is proposed to support process decomposition and knowledge reasoning. A matching algorithm is designed to detect instance constraints. The analysis indicates that the approach effectively realizes knowledge sharing, reuse and reasoning for context awareness applications in sensor networks.

\section{Introduction}

Context is defined as any information that can be used to characterize the situation of an entity. Context awareness refers to the idea that computers can both sense, and react based on their environment[1]. With the development of miniaturization and low power consumption of computer hardware devices and improvements of high bandwidth network technologies, computing capability is embedded into various sensor devices to achieve real-time processing and online-service. In this context, the traditional computing model is extended to the sensor network environment. Sensor device may have information about the circumstances under which they are able to operate and based on rules, or an intelligent stimulus, react accordingly.

Ontology is a formal definition and naming of the types, properties, and interrelationships of the entities that exist for a particular domain of discourse[2]. With its standardization, formalization, rich semantic expression ability and excellent reasoning ability, ontology can effectively solve the key problems such as formal representation, knowledge sharing and reuse, interoperating and context reasoning. Ontology is recognized as one of the promising technologies in the field of context aware computing. In sensor networks, context awareness-based service systems play more important roles. Since ontology-oriented architecture can integrate sensing and reasoning, it has been the dominating architecture in sensor networks information services.

Park proposed smart city sensor cloud framework which provides ontology-enabled semantic reasoning scheme. The framework enhances the availability and interoperability of the contextual information[3]. Kim developed an ontology-based healthcare context information model to implement a ubiquitous environment. Application developers can use the sensed information based on this common ontology[4]. Nalepa presented an overview of common approaches for modeling context, and proposed a rule-based context reasoning platform tailored to the needs of intelligent distributed mobile computing devices[5]. Fernandez presented a system for ontology alignment in the semantic sensor Web which uses fuzzy logic techniques to combine similarity measures between entities of different ontologies[6]. Roda presented an ontology-based framework for intelligent data analysis. The framework is based on a knowledge model composed by three ontologies which have been aligned through DOLCE Ultra-Lite upper ontology[7].

The above researches effectively improve the efficiency of context awareness system development for sensor networks, but a key problem for further study is how to provide build a generic and extensible context model to improve the validity and timeliness of the context reasoning. 
This paper proposes architecture for designing and developing context awareness systems for sensor networks. Our architecture supports the following features: A common sensor ontology model is proposed for sensor networks context awareness technology domain. In terms of context awareness process modeling, we use process decomposition technology for knowledge reasoning.

\section{Sensor Networks Ontology}

Ontology describes concepts and relationships of objective world, and implements knowledge sharing and reuse. Ontology is adopted to formally express the shared concepts and the relations among concepts in a domain, it has three meanings. First, the shared concepts include conceptual framework for domain knowledge modeling, interoperability protocol, and common agreement on basic theory of specific domain. Second, description of ontology is formal, and supports the reasoning for domain concepts and relations. Finally, ontology must be consistent, that is, there aren't logically contradictory statements or reasoning between the concepts and the relations.

Definition 1 Concept. Concept is abstract description of object in objective world, and is generally expressed as a collection of things, functions, actions, processes, and strategies. Formally, concept is represented as a four-tuple $\mathrm{C}=\{\mathrm{ID}, \mathrm{V}, \mathrm{SP}, \mathrm{IC}\}$, where ID is a unique identifier of concept, we denote ID as universal resource identifier; V is vocabulary used by concept; SP is a set of basic properties of concept; and IC is a set of instances of concept.

Definition 2 Ontology. Ontology is a formal and consistent description of domain sharing concept. Formally, ontology is represented as a six-tuple $\mathrm{O}=\{\mathrm{C}, \mathrm{R}, \mathrm{HC}, \mathrm{HR}, \mathrm{A}, \mathrm{I}\}$, where $\mathrm{C}$ is a set of domain concept; $\mathrm{R}$ is a relation set of concept; $\mathrm{HC}$ establishes hierarchical structure model on concepts, both $\mathrm{HC}$ and $\mathrm{R}$ are the subsets of Cartesian product of concept set; HC establishes hierarchical structure model on relations; A and I are the set of axioms and of ontology instances, respectively.

By the above definitions, the concepts of objective world, the relations among concepts, the concept classifications, and the objective laws are mapped to the concept sets of the ontology base, the relations and their hierarchical structures, the concept-hierarchy structure sets as well as the axioms. In this way, the objective world can be mapped to multiple formal sets.

The method of ontology-based knowledge representation is as follows. By means of ontological concept and knowledge description language, knowledge is represented and organized reasonably and effectively. Therefore, knowledge in sensor ontology-based context awareness domain is a kind of structured knowledge, and can be represented by the following model. The concept of context awareness is used as the smallest element of knowledge representation, and its features can be expressed as the attributes of ontology concepts. The hierarchical structure of ontology concepts is used as a vertical connection of domain knowledge. Similarly, the relations between ontology concepts are as horizontal connections. Domain axioms are used as constraints for perception procedures. In the way, we can represent context-aware domain knowledge for sensor networks.

\section{Sensor Networks Ontology Modeling}

In the physical world, context awareness can take place directly. In sensor networks, we hope to achieve indirect interaction with physical sensor device(PSD). That is, by way of calling, we interact with PSDs to obtain information about PSDs, or to control services provided by PSDs. In the digital world, PSD is represented by virtual sensor device(VSD). VSD has the following basic characteristics. Firstly, a PSD may have a plurality of corresponding VSDs, each VSD has one and only one unique identifier. Secondly, VSD can be classified into two categories: active and inactive VSD, active VSDs can run applications to access other services, resources, and agents. Inactive VSD is a passive software element, such as a database entry or other digital representation of physical sensor device. Thirdly, a group of given attributes of physical sensor device are expressed synchronously by VSD. This means that relevant digital parameters representing the characteristics of PSDs will be updated with the change of VSDs. In the same way, the changes of PSDs will also affect the corresponding VSDs. 
Resources not only refer to environmental information which is sensed by PSDs, but also refer to software components capable of manipulating PSDs. Resources usually provide external interfaces. Resources in sensor networks are divided into two categories: PSD resources and networks resources. There are some differences between these two types of resources. PSD resources are hosted on PSDs, that is, software systems are deployed on local device which attach to PSDs. PSD resources includes both executable codes for accessing, processing, and storing sensor information, and codes for controlling executor. Network resources are the resources that are available in sensor networks. Back-end database, for example, belongs to network resources. VSDs are also related to resources. By using resources, we can interact with VSDs corresponding to PSDs.

Services provide well-defined, standardized interfaces, as well as all the necessary functions to interact with PSDs. Interactions between services are realized by sensor networks. Services make sure resources can be accessed. Relations between resources and VSDs are modeled as associations between services and VSDs. Services are divided into three different abstract levels: the resource level, the VSD level, and the integration level. By accessing managed resources, resource-level service exposes VSD's functions. At the same time, the kind of services also deals with managing non-functional aspects such as reliability, security, resilience, and performance. VSD-level service provides interfaces for accessing VSD-level information. Integration-level service is composed of resource-level service, VSD-level service, and arbitrary combination of them.

Sensor networks should support mechanisms that enable them to respond automatically to events that are taking place either inside or outside application systems. Rule is a short-cut for referring to structure of active rule in event driven architecture, and can be used to perceive VSD contextual information. Such rule consists of three parts. Event part specifies signal that triggers rule invocation. Condition part is a logical test, if test is met, actions are carried out. Action part consists of updates or invocations on services. Fig.1 shows ontology concepts and their relations used in sensor networks context awareness technology domain. The domain consists of three subdomains, such as process domain, VSD domain and reasoning domain.

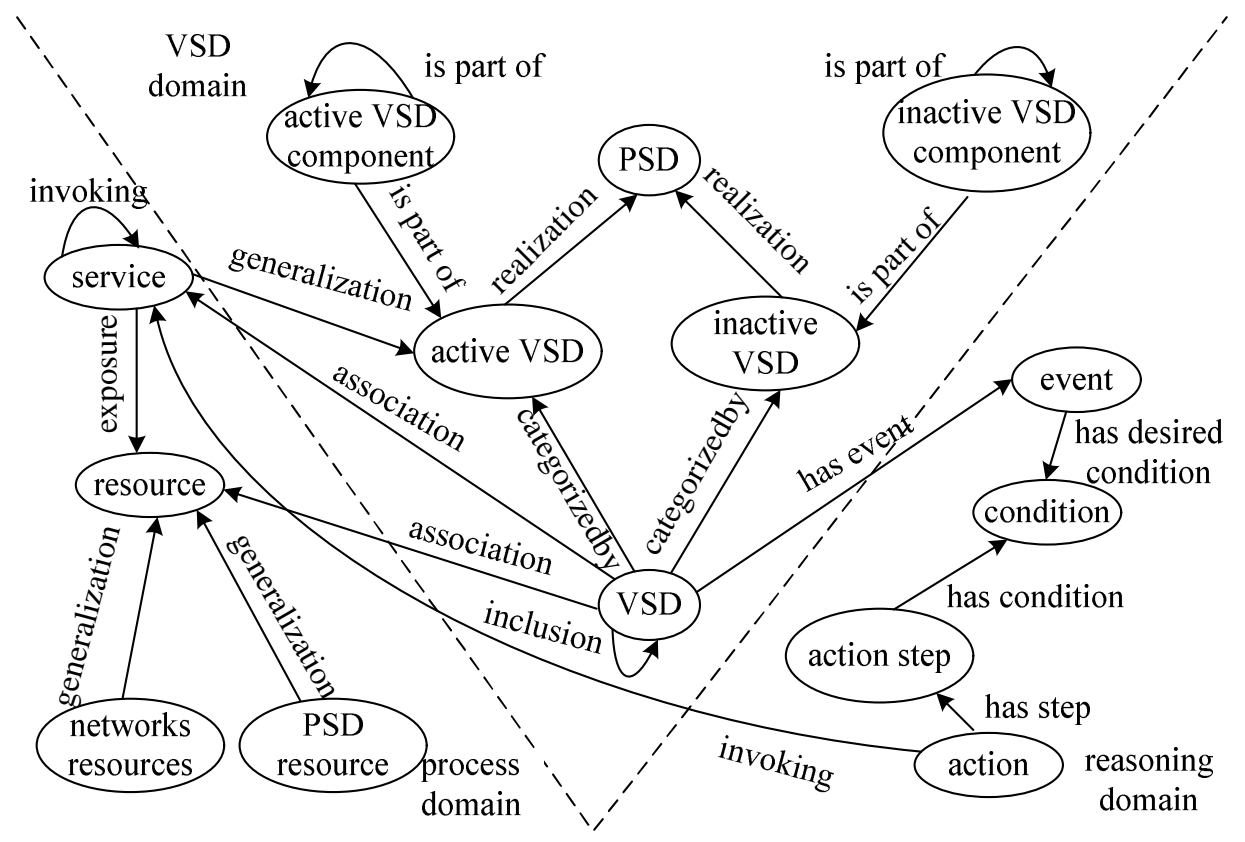

Fig.1. Ontology concepts and their relations

\section{Context Awareness Process Modeling}

Context awareness process decomposition. After establishing ontology model of sensor networks, we need to use process modeling techniques to identify and decompose key context awareness processes, and extract the semantic of data, function, organization and process generated in the process of context awareness. We divide the process into a series of steps and state transitions, 
and focus on unified expressions of process knowledge and semantic associations. Context awareness process can be decomposed into the following processes, such as preconditions, actions, results and related executions. Action refers to behavior sequence unit of sensor networks. If precondition is met, action is applied to target VSDs, and thus generates results of executing context awareness process. Basic elements of process are combined into a basic model of context awareness behavior steps and their logical environment. Basic elements obtained after decomposition correspond to the ontologies and relations in process domain. Context awareness process can be viewed as a set of process step instances. Each process step consists of multiple states.

Knowledge reasoning. Mapping relations from VSD state space to context awareness feature space are expressed as ontology mapping relations from VSD running states to context awareness signs. The essence of context awareness in sensor networks is as follows. According to the mapping relations from sign space to feature space, the matching of signs and context awareness features is conducted. In terms of complex context awareness objects, there is a complex relation between signs and context awareness features. The signs and context awareness features matching converts complex relation into the confidence level of signs. In the form of context awareness case base, the confidence level is integrated into the reasoning domain ontology. First, the matching algorithm is executed for instance constraint detection. Second, according to subordinate relations between signs and context awareness features, signs are matched with context awareness features in context awareness case base. In most cases, it is difficult to get all signs at once in the actual knowledge reasoning process. Based on the preliminary analysis to the surface signs, the matching algorithm proposes a possible feature set. The algorithm excludes the features which are not matched with the signs. The results are approaching to the context awareness results. When all signs appear on a context awareness object, it can be considered that the context awareness object is a final solution.

\section{Conclusion}

In this paper, we propose a semantic-oriented approach for architecting sensor networks applications using ontology mechanism. We derive conclusions of sensor networks ontology model and context awareness process model from analysis, and design a matching algorithm from two aspects: instance constraint detections, and subordinate relations between signs and context awareness features. The results show that the approach effectively realizes knowledge sharing, reuse and reasoning for context awareness applications in sensor networks.

\section{Acknowledgement}

This work was sponsored by Qing Lan project(Jiangsu province, china).

\section{References}

[1] G.D.Abowd, A.K.Dey, P.J.Brown, et al.: Towards a better understanding of context and context-awareness (1999).

[2] M.Baldauf, S.Dustdar, F.Rosenberg: International Journal of Ad Hoc and Ubiquitous Computing, Vol.2(4) (2007), p.263-277.

[3] K.Park, Y.Kim, J.Chang: International Journal of Distributed Sensor Networks, (2014).

[4] J.Kim, K.Y.Chung: Multimedia Tools and Applications, Vol.71(2) (2014), p.873-888.

[5] G.J.Nalepa, S.Bobek: Computer Science and Information Systems, Vol.11(1) (2014), p.171-193.

[6] S.Fernandez, I.Marsa-Maestre, J.R.Velasco, et al.: Sensors, Vol.13(9) (2013), p.12581-12604.

[7] F.Roda, E.Musulin: Expert Systems with Applications, Vol.41(17) (2014), p.7914-7926. 